TRANSACTIONS OF THE

AMERICAN MATHEMATICAL SOCIETY

Volume 349, Number 6, June 1997, Pages 2341-2358

S $0002-9947(97) 01940-5$

\title{
UNRAMIFIED COHOMOLOGY AND WITT GROUPS OF ANISOTROPIC PFISTER QUADRICS
}

\author{
R. SUJATHA
}

Dedicated to my father on his sixtieth birthday

\begin{abstract}
The unramified Witt group of an anisotropic conic over a field $k$, with char $k \neq 2$, defined by the form $\langle 1,-a,-b\rangle$ is known to be a quotient of the Witt group $W(k)$ of $k$ and isomorphic to $W(k) /\langle 1,-a,-b, a b\rangle W(k)$. We compute the unramified cohomology group $H_{n r}^{3} k(C)$, where $C$ is the three dimensional anisotropic quadric defined by the quadratic form $\langle 1,-a,-b, a b,-c\rangle$ over $k$. We use these computations to study the unramified Witt group of $C$.
\end{abstract}

Let $k$ be a field of characteristic not two and $K / k$ be a finitely generated field extension. The $n$-th unramified cohomology group of $K$ over $k$ with coefficients in $\mathbb{Z} / 2$ (cf. [CT]) is denoted $H_{n r}^{n}(K / k)$ (or just $H_{n r}^{n}(K)$ when the ground field being considered is clear). It is a subgroup of the Galois cohomology group $H^{n}(K, \mathbb{Z} / 2)$ (abbreviated to $\left.H^{n}(K)\right)$ and is defined as

$$
H_{n r}^{n}(K / k)=\bigcap_{v \in \mathcal{V}(K)} H_{e t}^{n}\left(\operatorname{Spec} \mathcal{O}_{v}, \mu_{2}\right) .
$$

Here $\mathcal{V}(K)$ is the set of all rank one discrete valuations on $K$ that are trivial on $k$. For $v \in \mathcal{V}(K), \mathcal{O}_{v}$ denotes the corresponding discrete valuation ring; it is well-known that the group $H_{e t}^{n}\left(\operatorname{Spec} \mathcal{O}_{v}, \mu_{2}\right)$ injects into $H^{n}(K)$. If $X / k$ is a smooth projective geometrically integral variety and $k(X)$ is its function field, then - by abuse of terminology - we refer to the groups $H_{n r}^{n}(k(X))$ as the unramified cohomology groups of $X$.

For a field $F$, let $W(F)$ be the Witt group of quadratic forms over $F$ [Sc]. For $k$ and $K / k$ as above, we can similarly define the unramified Witt group of $K / k$, denoted $W_{n r}(K / k)$, as the subgroup of $W(K)$ defined by

$$
W_{n r}(K / k)=\bigcap_{v \in \mathcal{V}(K)} W\left(\mathcal{O}_{v}\right)
$$

Here $W\left(\mathcal{O}_{v}\right)$ is the Witt group of the discrete valuation ring $\mathcal{O}_{v}$, which is a subgroup of $W(K)$ [Sc, Chapter VI]. If $X / k$ is smooth projective, then we refer to $W_{n r}(k(X))$ as the unramified Witt group of $X$.

Recall that a variety $X$ is said to be $k$-rational if $X$ is $k$-birational to the projective space. Then the natural maps $H^{n}(k) \rightarrow H^{n}(k(X))($ resp. $W(k) \rightarrow W(k(X)))$ induce isomorphisms $H^{n}(k) \simeq H_{n r}^{n}(k(X))$ (resp. $\left.W(k) \simeq W_{n r}(k(X))\right)$. Thus the unramified cohomology groups of a $k$-rational variety (resp. the unramified Witt

Received by the editors November 7, 1995.

1991 Mathematics Subject Classification. Primary 11E70; Secondary 13K05, 12G05.

Key words and phrases. Pfister forms, unramified cohomology, étale cohomology. 
group) coincide with the Galois cohomology groups of $k$ (resp. the Witt group of $k)$. In particular, this is true for smooth projective quadrics $X / k$ such that $X$ has a $k$-rational point. A natural question, therefore, is the nature of these groups when $X / k$ is a smooth projective quadric with no $k$-rational point.

For any field $F$, let $\left\langle\left\langle a_{1}, a_{2}, . ., a_{n}\right\rangle\right\rangle$ denote the $n$-fold Pfister form $\left\langle 1,-a_{1}\right\rangle \otimes$ $\left\langle 1,-a_{2}\right\rangle \otimes \cdots \otimes\left\langle 1,-a_{n}\right\rangle$. For $a \in F^{*}$, let $(a)$ denote its class in $H^{1}(F) \simeq F^{*} / F^{*^{2}}$. Given $\left(a_{1}\right), \ldots\left(a_{n}\right)$ in $H^{1}(F)$, let $\left(a_{1}\right) \cup \cdots \cup\left(a_{n}\right)$ denote the element in $H^{n}(F)$ obtained by taking cup-products. In this paper, we compute the unramified cohomology group $H_{n r}^{3}(k(C))$, where $C$ is a smooth projective anisotropic quadric of dimension three defined by the quadratic form $\langle\langle a, b\rangle\rangle \perp\langle-c\rangle$ over $k$. We prove that the group $H_{n r}^{3}\left(k(C)\right.$ ) (cf. $\S 1$ ) is a quotient of $H^{3}(k)$ if $(a) \cup(b) \cup(c) \cup(-1)$ is not trivial in $H^{4}(k)$. If the element $(a) \cup(b) \cup(c) \cup(-1)$ is trivial in $H^{4}(k)$, then we show that the natural map $H^{3}(k) \rightarrow H_{n r}^{3}(k(C))$ has cokernel $\mathbb{Z} / 2$. We also prove a "descent" result for special symbols in $H_{n r}^{3}(k(C))$. More precisely, suppose $\theta \in\left[(a) \cup(b) \cup H^{1}(k(C))\right] \cap H_{n r}^{3}(k(C))$. We then show that $\theta$ is "extended" from $H^{3}(k)$ i.e. $\theta=(a) \cup(b) \cup(e)$, for some $(e) \in H^{1}(k)$. For special ground fields $k$, (e.g. $k$ a global field), we compute higher unramified cohomology groups of $C$.

These results are then used to study the unramified Witt group of the quadric $C$. For anisotropic conics over $k$, it is a result of Parimala [P, Theorem 4.2] that the unramified Witt group is a quotient of $W(k)$. This result was extended by ColliotThélène and the author to real anisotropic quadrics of dimension three [CT-S]. It was shown that if $C / \mathbb{R}$ is defined by $\langle\langle-1,-1\rangle\rangle \perp\langle 1\rangle$, then $W_{n r}(\mathbb{R}(C)) \simeq$ $W(\mathbb{R}) /\langle\langle-1,-1,-1\rangle\rangle$. In this paper, we consider the case when $k$ is a global field which is not totally imaginary. (Note that over a totally imaginary global field, any quadratic form of dimension five or more is isotropic.)

Suppose $k$ is a global field as above. To transfer the results obtained in cohomology to Witt groups, we need to compare the quotients of the induced filtration on $W_{n r}(k(C))$ with the unramified cohomology groups. In order to do this, we need Rost's announcement that the norm residue homomorphism in degree four is an isomorphism and the map $e_{5}$ (cf. $\S 1$ ) is well-defined. One can then use results of Arason-Elman-Jacob to carry out the above mentioned comparison. Using Jannsen's higher dimensional Hasse principle, we see that the induced filtration on the unramified Witt group is finite. One then deduces that $W_{n r}(k(C)) \simeq W(k) /\langle\langle a, b, c\rangle\rangle$. This has the interesting corollary that any unramified quadratic form over $k(C)$ is in fact defined over $k$.

Note that it is not in general true that the unramified Witt group of an arbitrary anisotropic quadric over a field $k$ is a quotient of $W(k)$. Bruno Kahn [Ka] has constructed an example of an unramified Witt class that is not in the image of $W(k)$ for an anisotropic "Albert quadric", i.e. a quadric defined by an Albert form over $k$. However, we wonder if the unramified Witt group of "Pfister quadrics" over $k$ (i.e. quadrics defined by Pfister forms and their neighbours) is a quotient of $W(k)$ for arbitrary fields $k$. We show that this is indeed the case for a quadric defined by three-fold Pfister forms if the following statement is true:

(A): The subgroup $[\langle\langle a, b\rangle\rangle I(k(C))] \cap I_{n r}^{3}(k(C))$ is generated by elements of the form $\langle\langle a, b, f\rangle\rangle \in I_{n r}^{3}(k(C))$, where $I_{n r}^{3}(k(C))=W_{n r}(k(C)) \cap I^{3}(k(C))$.

Here, $I^{n}(k(C))$ denotes the natural filtration on the Witt group (cf. $\left.\S 1\right)$ and $I_{n r}^{n}(k(C))$ denotes the induced filtration on the unramified Witt group $W_{n r}(k(C))$.

The paper consists of four sections. In section 1, we list various results and notation that will be used in the later sections. Section 2 contains relevant results 
on the unramified cohomology groups of the quadric $C$ and some of its subgroups. In section 3 , we specialise to the case when the ground field $k$ is a global field. These results are used in section 4 to study the unramified Witt group $W_{n r}(k(C))$.

We would like to thank M. Szyjewski for helpful discussions and Bruno Kahn for pointing out a gap (namely assuming that (A) is true!) in an earlier version of the paper. We would also like to thank Uwe Jannsen for his notes on the higher dimensional Hasse principle. Finally, the partial support of NATO grant CRG 940307 is gratefully acknowledged.

Throughout the paper, the ground field $k$ is assumed to be of characteristic not 2. For a more detailed exposition of various preliminary results, we refer the reader to $\S \S 1$ and 2 of [CT-S], and to Scharlau's book [Sc] for all results and terminology from quadratic form theory.

\section{Preliminary Results}

In this section, we list various results that will be needed in the later sections.

1.1. Unramified cohomology and the Bloch-Ogus spectral sequence. Let $X / k$ be a smooth, geometrically integral variety and let $k(X)$ be its function field. For any variety $Y$ over $k$, we denote the étale cohomology groups $H_{e t}^{n}\left(Y, \mu_{2}\right)$ of $Y$ with coefficients in the étale sheaf $\mu_{2}$ of square roots of unity by $H^{n}(Y)$. Let $\mathcal{H}^{q}$ be the Zariski sheaf associated to the presheaf $U \mapsto H^{n}(U)$, where $U$ is Zariski open in $X$. By the results of Bloch-Ogus [B-O], the group of global sections of the sheaf $\mathcal{H}^{q}$, denoted $H^{0}\left(X, \mathcal{H}^{q}\right)$, is a subgroup of the Galois cohomology group $H^{q}(k(X))$. Here, for a field $F$ with $\operatorname{char} F \neq 2, H^{n}(F):=H^{n}\left(\operatorname{Gal}\left(F_{s} / F\right), \mathbb{Z} / 2\right)$ is the Galois cohomology group with coefficients in $\mathbb{Z} / 2$, and $F_{s}$ denotes a separable closure of $F$. Further, if $X$ is proper, the group $H^{0}\left(X, \mathcal{H}^{q}\right)$ is a birational invariant of $X[\mathrm{CT}-\mathrm{O}$, Remarque 1.1.3] and is also denoted by $H_{n r}^{q}(k(X))$. These groups are called the unramified cohomology groups of $X$ (cf. [CT-O, 1.1.1]). For instance, if $X$ is smooth and proper, the group $H_{n r}^{2}(k(X))=H^{0}\left(X, \mathcal{H}^{2}\right)$ is the group of 2-torsion elements in the Brauer group of $X$. We will often use the notation $H_{n r}^{q}(k(X))$ or $H^{0}\left(X, \mathcal{H}^{q}\right)$ to denote the unramified cohomology groups in the case that $X$ is smooth and proper.

Let $H^{p}\left(X, \mathcal{H}^{q}\right)$ denote the Zariski cohomology groups of $\mathcal{H}^{q}$. Then there is a spectral sequence $[\mathrm{B}-\mathrm{O}]$

$$
E_{2}^{p, q}=H^{p}\left(X, \mathcal{H}^{q}\right) \Longrightarrow H^{n}(X)=E^{n} .
$$

The filtration on $H^{n}(X)$ coincides with the filtration given by the codimension of support. Further, it follows from the Bloch-Ogus resolution of the sheaf $\mathcal{H}^{q}$ [B-O, Theorem 4.2] that $H^{p}\left(X, \mathcal{H}^{q}\right)=0$ for $p>q$. Another well-known result that we will need is the isomorphism [B-O, Theorem 7.7]

$$
H^{p}\left(X, \mathcal{H}^{p}\right) \simeq C H^{p}(X) / 2
$$

where $C H^{p}(X)$ denotes the Chow group of codimension $p$ cycles on $X$ modulo rational equivalence. The spectral sequence (1) thus gives an exact sequence

$$
\cdots \rightarrow H^{3}(X) \rightarrow H^{0}\left(X, \mathcal{H}^{3}\right) \stackrel{d}{\rightarrow} C H^{2}(X) / 2 \rightarrow H^{4}(X) \rightarrow \cdots .
$$

The map $C H^{2}(X) / 2 \rightarrow H^{4}(X)$ is in fact the cycle map (cf. [CT-S, $\left.\S 1\right]$ ). 
1.2. Galois cohomology, quadratic forms and function fields of quadrics. We recall some results from quadratic form theory and Galois cohomology. Given a field $F$, char $F \neq 2$, let $H^{n}(F)$ denote the $n$-th Galois cohomology group. Let $W(F)$ denote the Witt group of quadratic forms over $F$. There is a standard filtration on $W(F)$, denoted $\left(I^{n}(F)\right)_{n \geq 0}$, where $I(F)$ is the fundamental ideal of even dimensional forms and $I^{n}(F)$ its $n$-th power. Recall that the set $P^{n}(F)$ of $n$ fold Pfister forms additively generates $I^{n}(F)$. There are maps $e_{n}: P^{n}(F) \rightarrow H^{n}(F)$ which send an $n$-fold Pfister form $\left\langle\left\langle a_{1}, a_{2}, \ldots, a_{n}\right\rangle\right\rangle$ to the element $\left(a_{1}\right) \cup\left(a_{2}\right) \cup \ldots \cup$ $\left(a_{n}\right)$ [AEJ]. These maps are known to extend to well-defined homomorphisms on $I^{n}(F)$ for all fields $F$ when $n \leq 4[\mathrm{~A}],[\mathrm{J}-\mathrm{R}],[\mathrm{Sz}]$. Further, the maps

$$
\bar{e}_{n}: I^{n}(F) / I^{n+1}(F) \rightarrow H^{n}(F)
$$

are known to be isomorphisms for $n \leq 3$ [Me], [Me-S1], [R]. Rost has announced a proof that $\bar{e}_{4}$ is an isomorphism and that $e_{5}$ is well-defined [R1]. If the maps $e_{i}$ are well-defined homomorphisms for all fields $F$ and for $i \leq m$, then it was observed by Parimala $[\mathrm{P} 2, \S 1]$, that one can compare the filtrations on the unramified Witt group with the unramified cohomology groups. Indeed, there are homomorphisms $I_{n r}^{m}(F) \rightarrow H_{n r}^{m}(F)$ induced by the homomorphisms $e_{m}$, where $F$ is now a finitely generated extension of the ground field $k$.

We mention the following results about function fields of quadrics. Let $Q / k$ be the smooth projective quadric defined by an $n$-fold Pfister form $\left\langle\left\langle a_{1}, a_{2}, \ldots, a_{n}\right\rangle\right\rangle$ over $k$ and let $k(Q)$ be the function field. The following conjecture is referred to as Conjecture $A_{n}$ :

$$
\operatorname{Ker}\left(H^{n}(k) \rightarrow H^{n}(k(Q))\right)=\left\{0,\left(a_{1}\right) \cup\left(a_{2}\right) \cup \cdots \cup\left(a_{n}\right)\right\} .
$$

This conjecture is known to be true for $n \leq 4$. Rost [R1] has announced a proof for $n=5$. The cases $n=1,2$ are classical, the case $n=3$ was proved by Arason [A, Satz 5.4] and the case $n=4$ was proved by Jacob-Rost [J-R] and independently, by Szyjewski $[\mathrm{Sz}]$.

For Witt groups, we have an analogous result, namely $[\mathrm{A}]$, [Pf]

$$
\operatorname{Ker}\left(W(k) \rightarrow W(k(Q))=\left\langle\left\langle a_{1}, a_{2}, \ldots, a_{n}\right\rangle\right\rangle W(k) .\right.
$$

Finally, recall that a form $\psi$ over $F$ is said to be a Pfister neighbour of an $n$-fold Pfister form $\phi$ over $F$ if $\operatorname{dim} \psi \geq 2^{n-1}+1$ and $\psi$ is isometric to a subform of $\phi$. Let $\phi$ be an $n$-fold Pfister form and $\psi$ a Pfister neighbour of $\phi$. We denote the quadrics defined by $\phi$ and $\psi$ by $X_{\phi}$ and $X_{\psi}$ respectively. Let $F\left(X_{\phi}\right)$ (resp. $F\left(X_{\psi}\right)$ ) denote the corresponding function fields. We record the following results for later use. We have [J-R, Lemma 1.2] for $m \geq 0$,

$$
\operatorname{Ker}\left(H^{m}(F) \rightarrow H^{m}\left(F\left(X_{\phi}\right)\right)\right)=\operatorname{Ker}\left(H^{m}(F) \rightarrow H^{m}\left(F\left(X_{\psi}\right)\right)\right)
$$

and

$$
\operatorname{Ker}\left(W(F) \rightarrow W\left(F\left(X_{\phi}\right)\right)\right)=\operatorname{Ker}\left(W(F) \rightarrow W\left(F\left(X_{\psi}\right)\right)\right) .
$$

(This follows from the observation that $X_{\phi}$ has a $k\left(X_{\psi}\right)$-rational point (and viceversa), namely the generic point. Thus the field $k\left(X_{\phi}\right)\left(X_{\psi}\right)$ is a purely transcendental extension of both $k\left(X_{\phi}\right)$ and $k\left(X_{\psi}\right)$.) We record that if $m=2$ (resp. $m=3$ ) and $\phi=\langle\langle a, b\rangle\rangle, a, b \in F^{*}$ (resp. $\left.\phi=\langle\langle a, b, c\rangle\rangle, a, b, c \in F^{*}\right)$, then the kernel in (6) is the subgroup consisting of elements of the form $\left\{(a) \cup(b) \cup H^{1}(F)\right\}[\mathrm{A}]$ (resp. $\left.\left\{(a) \cup(b) \cup(c) \cup H^{1}(F)\right\}[\mathrm{J}-\mathrm{R}][\mathrm{Sz}]\right)$. 
1.3. Chow groups and étale cohomology groups of quadrics. We now recall some results of Karpenko, Merkurjev and Szyjewski. Let $C / k$ be the smooth projective anisotropic quadric of dimension three, defined by the anisotropic quadratic form $\langle\langle a, b\rangle\rangle \perp\langle-c\rangle$ over $k$. Thus $C \subset \mathbb{P}_{k}^{4}$ is defined by $X_{0}^{2}-a X_{1}^{2}-b X_{2}^{2}+a b X_{3}^{2}-$ $c X_{4}^{2}=0$. Let $B$ be the hyperplane section of $C$ defined by $X_{4}=0$ and let $X$ be the plane section of $C$ defined by $X_{4}=0, X_{3}=0$. Then $[X]$ is a cycle of codimension one (respectively of codimension two) in $B$ (resp. in $C$ ). Let $\left[T_{1}\right]$ be the double line cycle (of codimension one in $B$ and codimension two in $C$ ), defined by the equations $X_{0}^{2}-a X_{1}^{2}=X_{0} X_{3}-X_{1} X_{2}=X_{4}=0$. It is known (cf. [K-Me, 1.8]) that $C H^{1}(B) \simeq \operatorname{Pic}(B) \simeq \mathbb{Z}[X] \oplus \mathbb{Z}\left[T_{1}\right]$ and that $C H^{2}(C) \simeq \mathbb{Z}\left[T_{1}\right] \oplus \mathbb{Z} / 2\left[X-T_{1}\right]($ cf. $[\mathrm{Sz}$, Proposition 3.3.6]). The natural map

$$
g: C H^{1}(B) \rightarrow C H^{2}(C)
$$

maps the class $\left[T_{1}\right]$ to the same class considered now as an element of $C H^{2}(C)$ and similarly $\left[X-T_{1}\right]$ to its corresponding class, which is now the 2-torsion element in $C H^{2}(C)$. Thus the map $g$ is surjective and the induced map

$$
\bar{g}: C H^{1}(B) / 2 \rightarrow C H^{2}(C) / 2
$$

is an isomorphism, being a surjective map between two spaces of the same dimension. It is clear from the classical localisation sequence (cf. [K-Me, Proposition $2.3])$ and (2) that the groups $\operatorname{Pic}(V)$ and $C H^{2}(V)$ are trivial, where $V$ is the open set $C \backslash B$. Further, the cycle class map $c l_{B}: C H^{1}(B) / 2 \rightarrow H^{2}(B)$ is injective. The images of the generators are denoted by $c l_{B}[X]$ and $c l_{B}\left[X-T_{1}\right]$. The images under the cycle map $c l_{C}: C H^{2}(C) / 2 \rightarrow H^{4}(C)$ of the elements $\left[T_{1}\right]$ and $\left[X-T_{1}\right]$ are denoted by $c l_{C}\left[T_{1}\right]$ and $c l_{C}\left[X-T_{1}\right]$. We have the commutative diagram below (cf. [CT-S, §1], observing that the statements there are valid for a general field of char $\neq 2)$ :

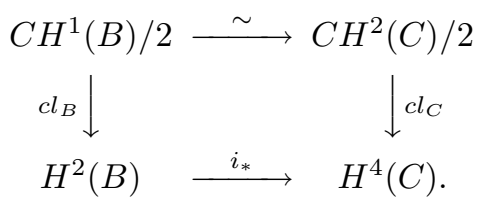

Szyjewski proved [Sz, Corollary 5.3.4] that the element $\mathrm{cl}_{C}\left[T_{1}\right]=(a) \cup(b) \cup c l_{C}[B]$, where $c l_{C}[B]$ is the image in $H^{2}(C)$ of the non-trivial element under the Gysin map $H^{0}(B) \rightarrow H^{2}(C)$ and $(a) \cup(b) \in H^{2}(k) \hookrightarrow H^{2}(C)$ [Sz, Lemma 5.2.1 (b)]. Further, $c l_{C}\left[X-T_{1}\right] \in H^{4}(k)$ and generates the kernel subgroup $\operatorname{Ker}\left(H^{4}(k) \rightarrow H^{4}(V)\right)[\mathrm{Sz}$, Corollary 5.3.4], where $V$ is the open set $C \backslash B$.

\section{UNRAMIFIED COHOMOLOGY AND ÉTALE COHOMOLOGY}

In this section, we use the results of Szyjewski and compute the structure of the unramified cohomology group $H_{n r}^{3}(k(C))$. We continue with the notation in $\S 1$. We have

Theorem 1. Let $C / k$ be the smooth projective anisotropic quadric defined by the form $\langle\langle a, b\rangle\rangle \perp\langle-c\rangle$ over $k$. Let $k(C)$ be the function field of $C$ and $H_{n r}^{3}(k(C))$ denote the unramified cohomology group. Then

$$
H_{n r}^{3}(k(C)) \simeq H^{3}(k) /(a) \cup(b) \cup(c) \text { if }(a) \cup(b) \cup(c) \cup(-1) \neq 0 \text { in } H^{4}(k)
$$


and

$H_{n r}^{3}(k(C)) \simeq\left(H^{3}(k) /(a) \cup(b) \cup(c)\right) \oplus \mathbb{Z} / 2$ if $(a) \cup(b) \cup(c) \cup(-1)=0$ in $H^{4}(k)$.

Proof. Let $\left(H^{3}(C)^{(i)}\right)_{i \geq 0}$ denote the filtration on $H^{3}(C)$ given by the Bloch-Ogus spectral sequence (1.1). Then $H^{3}(C)^{(1)}=\operatorname{Ker}\left(H^{3}(C) \rightarrow H^{3}(k(C))\right)$. Using the Hochschild-Serre spectral sequence (cf. [Sz, Lemma 5.2.1 (a)]), we have

$$
H^{3}(C) \simeq H^{3}(k) \oplus H^{1}(k) \cup c l_{C}[B] .
$$

Note that the edge maps $H^{n}(k) \rightarrow H^{n}(C)$ in the Hochschild-Serre spectral sequence are in fact induced by the structure morphism. Clearly, the second summand in the direct sum maps to zero in $H^{3}(k(C))$. By [A], the group $\operatorname{Ker}\left(H^{3}(k) \rightarrow H^{3}(k(C))\right)=(a) \cup(b) \cup(c)$. Thus we have (cf. [Sz, Corollary $5.2 .2])$,

$$
H^{3}(C)^{(1)} \simeq(a) \cup(b) \cup(c) \oplus H^{1}(k) \cup c l_{C}[B] .
$$

Hence the cokernel of the natural inclusion $H^{3}(C)^{(1)} \hookrightarrow H^{3}(C)$ is given by

$$
H^{3}(C) / H^{3}(C)^{(1)} \simeq H^{3}(k) /(a) \cup(b) \cup(c) .
$$

On the other hand we have an exact sequence (1.1)

$$
\cdots H^{3}(C) \rightarrow H^{0}\left(C, \mathcal{H}^{3}\right) \rightarrow C H^{2}(C) / 2 \rightarrow H^{4}(C)
$$

where the map at the extreme left is the cycle map. We study the kernel of this map.

Assume that $(a) \cup(b) \cup(c) \cup(-1) \neq 0$ in $H^{4}(k)$. We claim then that the cycle map is injective. By [Sz, Corollary 5.3.4, Proposition 5.4.6], we have $(a) \cup(b) \cup(c) \cup$ $(-1)=\operatorname{cl}_{C}\left[X-T_{1}\right]$. Recall (1.3) that $C H^{2}(C) / 2 \simeq \mathbb{Z} / 2\left[T_{1}\right] \oplus \mathbb{Z} / 2\left[X-T_{1}\right]$ and $c l_{C}\left[T_{1}\right]=(a) \cup(b) \cup c l_{C}[B]$. By the Hochschild-Serre spectral sequence, $H^{4}(k) \oplus$ $H^{2}(k) \cdot c l_{C}[B]$ injects into $H^{4}(C)$ [Sz, Lemma 5.2.1 (c)]. Thus, considering this description of the cycle map $\mathrm{cl}_{C}$ on the generators, we see that $c l_{C}: C H^{2}(C) / 2 \rightarrow$ $H^{4}(C)$ is injective. Therefore by $(9)$, we see that $H^{3}(C)$ surjects onto $H^{0}\left(C, \mathcal{H}^{3}\right)=$ $H_{n r}^{3}(k(C))$. We thus have an isomorphism $H^{3}(C) / H^{3}(C)^{(1)} \simeq H^{0}\left(C, \mathcal{H}^{3}\right)$, and hence

$$
H_{n r}^{3}(k(C)) \simeq H^{3}(k) /(a) \cup(b) \cup(c) .
$$

We now consider the case $(a) \cup(b) \cup(c) \cup(-1)=0$ in $H^{4}(k)$. Then, since $c l_{C}\left[X-T_{1}\right]=(a) \cup(b) \cup(c) \cup(-1)$ [Sz, Proposition 5.4.6], we see that the cycle map $c l_{C}$ is not injective and that $\operatorname{Ker}\left(c l_{C}\right)$ is in fact isomorphic to $\mathbb{Z} / 2\left(c l_{C}\left[X-T_{1}\right]\right)$. Using the exact sequence (9) along with (8), we get an exact sequence

$$
0 \rightarrow H^{3}(k) /(a) \cup(b) \cup(c) \rightarrow H_{n r}^{3}(k(C)) \rightarrow \operatorname{Ker}\left(c l_{C}\right) \rightarrow 0 .
$$

Hence in this case $H_{n r}^{3}(k(C)) \simeq\left(H^{3}(k) /(a) \cup(b) \cup(c)\right) \oplus \mathbb{Z} / 2$, and the theorem is proved.

We highlight the following:

Corollary. Let $C / k$ be as above. Then the cycle map

$$
C H^{2}(C) / 2 \rightarrow H^{4}(C)
$$


is injective if and only if $(a) \cup(b) \cup(c) \cup(-1) \neq 0$ in $H^{4}(k)$. If $(a) \cup(b) \cup(c) \cup(-1) \neq$ 0 in $H^{4}(k)$, then the kernel is isomorphic to $\mathbb{Z} / 2\left(c_{C}\left[X-T_{1}\right]\right)$.

We now consider the subgroup of $H_{n r}^{3}(k(C))$ which consists of elements that are of the form $(a) \cup(b) \cup H^{1}(k(C))$. We prove that this subgroup in fact lies in the subgroup $H^{3}(k) /(a) \cup(b) \cup(c)$ of $H_{n r}^{3}(k(C))$ even when $(a) \cup(b) \cup(c) \cup(-1)$ is trivial in $H^{4}(k)$. We first need the following fact.

Lemma 2. Let $C, B$ be as above and $V$ be the open subvariety $C \backslash B$. Then the natural restriction map $H^{0}\left(C, \mathcal{H}^{3}\right) \rightarrow H^{0}\left(V, \mathcal{H}^{3}\right)$ is an isomorphism.

Proof. Clearly this map is injective. Further, the group $C H^{2}(V)$ is zero (1.3). Thus, in the Bloch-Ogus spectral sequence for $V$, we see that the map $H^{3}(V) \rightarrow$ $H^{0}\left(V, \mathcal{H}^{3}\right)$ is surjective. The Hochschild-Serre spectral sequence for $V$ gives an exact sequence (cf. [Sz, 5.4])

$$
0 \rightarrow H^{3}(k) \rightarrow H^{3}(V) \rightarrow H^{0}(k) \stackrel{d}{\rightarrow} H^{4}(k) \rightarrow H^{4}(V) .
$$

Szyjewski proves that the differential coincides with multiplication by $(a) \cup(b) \cup(c) \cup$ $(-1)$ [Sz, Corollary 5.3.4(b), Proposition 5.4.6]. The edge map $H^{3}(k) \rightarrow H^{3}(V)$ is in fact the one induced by the structure morphism. We consider the following cases:

Case (i). $(a) \cup(b) \cup(c) \cup(-1) \neq 0$ in $H^{4}(k)$.

By the remarks above, we see that the natural map $H^{3}(k) \rightarrow H^{3}(V)$ is indeed an isomorphism. Hence $H^{3}(k)$ surjects onto $H^{0}\left(V, \mathcal{H}^{3}\right) \subseteq H^{3}(k(C))$ with kernel $(a) \cup(b) \cup(c) \cup(-1)$.

Case (ii). $(a) \cup(b) \cup(c) \cup(-1)=0$ in $H^{4}(k)$.

In this case, by Theorem 1, the natural map $H^{3}(k) \rightarrow H_{n r}^{3}(k(C))=H^{0}\left(C, \mathcal{H}^{3}\right)$ has kernel $(a) \cup(b) \cup(c)$ and cokernel $\mathbb{Z} / 2$. Further, by our assumption on the nontriviality of $(a) \cup(b) \cup(c) \cup(-1)$, using the sequence (12), one sees that the natural map $H^{3}(k) \rightarrow H^{3}(V)$ has cokernel $\mathbb{Z} / 2$. Consider the commutative diagram

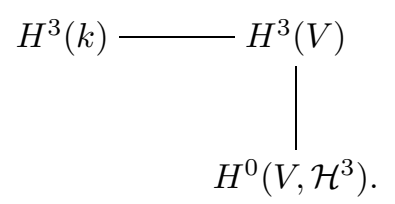

We have $\operatorname{Ker}\left(H^{3}(V) \rightarrow H^{0}\left(V, \mathcal{H}^{3}\right)\right)=\mathbb{Z} / 2$ [Sz, Lemma 5.4.3]. Thus we have $\operatorname{Ker}\left(H^{3}(k) \rightarrow H^{0}\left(V, \mathcal{H}^{3}\right)\right)=\operatorname{Ker}\left(H^{3}(k) \rightarrow H^{3}(k(C))\right)=(a) \cup(b) \cup(c)$ and the cokernel is $\mathbb{Z} / 2$. Hence the lemma is proved.

In order to prove that the subgroup of $H^{0}\left(C, \mathcal{H}^{3}\right)$ consisting of elements of the form $(a) \cup(b) \cup H^{1}(k(C))$ lies in the subgroup $H^{3}(k) /(a) \cup(b) \cup(c)$ of $H^{0}\left(C, \mathcal{H}^{3}\right)$, by the above lemma, it suffices to prove the same for $H^{0}\left(V, \mathcal{H}^{3}\right)$. In fact, we prove the stronger

Lemma 3. Let $C, V$ be as above. Then we have an inclusion

$$
\left[(a) \cup H^{2}(k(C))\right] \cap H_{n r}^{3}(k(C)) \subseteq H^{3}(k) /(a) \cup(b) \cup(c) .
$$


Proof. As mentioned above, we are reduced to proving

$$
\left[(a) \cup H^{2}(k(V))\right] \cap H^{0}\left(V, \mathcal{H}^{3}\right) \subseteq H^{3}(k) /(a) \cup(b) \cup(c) .
$$

Consider the quadratic extension $l=k(\sqrt{a})$ which splits $C$, and let $V_{l}$ be the variety $V \times{ }_{k} l$. Consider the following commutative diagram:

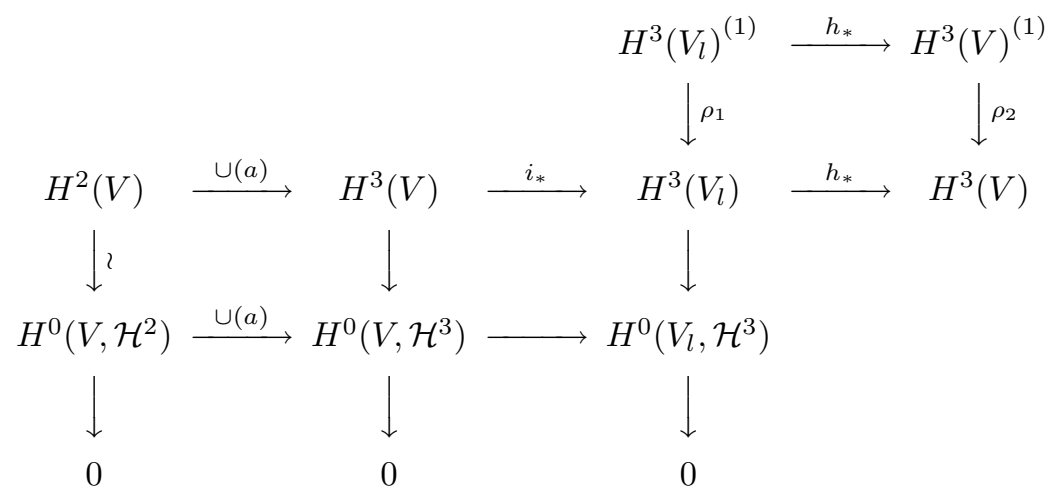

Here the middle row is exact and the columns are also exact. The vertical sequences are induced by the Bloch-Ogus spectral sequence and the maps $\rho_{1}$ and $\rho_{2}$ are the natural inclusions. The middle exact row is the long exact cohomology sequence associated to a quadratic extension of the ground field. The homomorphism $h_{*}$ is the transfer map, and the vertical arrow at the extreme left is an isomorphism since $\operatorname{Pic}(V) / 2 \simeq C H^{1}(V) / 2$ is zero. The map $H^{3}(V) \rightarrow H^{0}\left(V, \mathcal{H}^{3}\right)$ is surjective because $C H^{2}(V)$ is zero.

Suppose $(a) \cup(\zeta) \in H^{0}\left(V, \mathcal{H}^{3}\right)$, with $\zeta \in H^{2}(k(C))=H^{2}(k(V))$. Let $\theta \in H^{3}(V)$ be an element which maps to $(a) \cup(\zeta)$. There are two possibilities: either $i_{*}(\theta)=0$ in $H^{3}\left(V_{l}\right)$ or $i_{*}(\theta) \neq 0$ in $H^{3}\left(V_{l}\right)$. We consider these cases independently.

Case (i). Suppose $i_{*}(\theta)=0$ in $H^{3}\left(V_{l}\right)$. Then there is an element $\theta^{\prime} \in H^{2}(V)$ such that $\theta^{\prime} \cup(a)=\theta$. The natural map $H^{2}(k) \rightarrow H^{2}(V)$ is in fact an isomorphism. This is an easy consequence of the Hochschild-Serre spectral sequence, noting that the groups $H^{1}\left(V_{s}\right)$ and $H^{2}\left(V_{s}\right)\left(V_{s}=V \times k_{s}, k_{s}\right.$ a separable closure of $\left.k\right)$ are trivial. The latter statement is easily established from the long exact localisation sequence for the triple $B_{s} \hookrightarrow C_{s} \hookleftarrow V_{s}$. Hence we see that $\theta^{\prime} \in H^{2}(k)$ and $\theta=\theta^{\prime} \cup(a)$ in $H^{3}(k) \subseteq H^{3}(V)$. Now the result follows since $\theta$ maps to $(a) \cup(\zeta)$.

Case (ii). Suppose $i_{*}(\theta)$ is not zero in $H^{3}\left(V_{l}\right)$. We first observe that $(a) \cup(\zeta)$ maps to zero in $H^{0}\left(V_{l}, \mathcal{H}^{3}\right)$. This follows from the following commutative diagram:

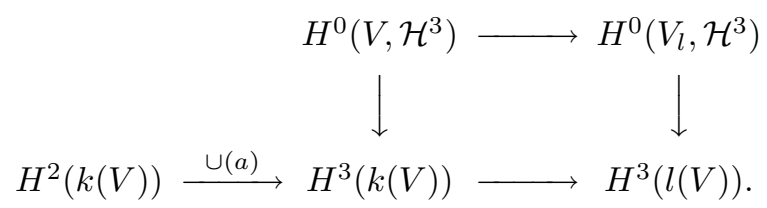

Here the vertical maps are inclusions and the bottom exact row is part of the long exact sequence associated to a degree two extension [A, Corollary 4.6]. Thus, we see that $i_{*}(\theta) \in H^{3}\left(V_{l}\right)^{(1)}$. By our assumption, $i_{*}(\theta)$ is not zero. Szyjewski proves that for both $V$ and $V_{l}$, the groups $H^{3}(V)^{(1)}$ and $H^{3}(V)_{l}^{(1)}$ are isomorphic to $\mathbb{Z} / 2$ [Sz, Lemma 5.4.2 (a)]. Further, the transfer map $H^{3}\left(V_{l}\right) \rightarrow H^{3}(V)$ induces an isomorphism $H^{3}\left(V_{l}\right)^{(1)} \simeq H^{3}(V)^{(1)}\left[\mathrm{Sz}\right.$, Lemma 5.4.3]. Thus we see that $i_{*}(\theta)=\alpha_{l}$ 
is the generator of $H^{3}\left(V_{l}\right)^{(1)}$ and $h_{*}\left(\alpha_{l}\right)=\alpha$, where $\alpha$ is the generator of $H^{3}(V)^{(1)} \simeq$ $\mathbb{Z} / 2$ [Sz, Lemma 5.4.3]. But $h_{*}\left(\alpha_{l}\right)=h_{*}\left(i_{*}(\theta)\right)=0$, since the middle row of (13) is exact. This is a contradiction and hence this case does not occur. Therefore the lemma is proved.

We refine the above lemma further.

Lemma 4. Let $C$ be as above. Then we have an isomorphism

$$
\left[(a) \cup(b) \cup H^{1}(k(C))\right] \cap H_{n r}^{3}(k(C)) \simeq(a) \cup(b) \cup H^{1}(k) /(a) \cup(b) \cup(c) .
$$

Proof. Consider the exact sequence (cf. (1.2))

$$
0 \rightarrow(a) \cup(b) \cup H^{1}(k(C)) \rightarrow H^{3}(k(C)) \stackrel{\bar{\phi}}{\rightarrow} H^{3}(k(C \times B))
$$

where $B$ is considered as a quadric defined over $k(C)$. Since $C$ has a rational point over $k(B)$, the field $k(C \times B)=k(B \times C)$ is a pure transcendental extension of $k(B)$. The unramified cohomology groups are invariant under pure transcendental extensions [CT-O, Proposition 1.2]. Hence, the restriction of the above exact sequence to unramified subgroups gives an exact sequence

$$
0 \rightarrow\left[(a) \cup(b) \cup H^{1}(k(C))\right] \cap H_{n r}^{3}(k(C)) \rightarrow H_{n r}^{3}(k(C)) \stackrel{\bar{\phi}}{\rightarrow} H_{n r}^{3}(k(B)) .
$$

Consider the commuting diagram below, where the maps $\phi_{C}$ and $\phi_{B}$ are the natural maps induced by the inclusions $k \hookrightarrow k(C)$ and $k \hookrightarrow k(B)$ respectively. We have

$$
\begin{aligned}
& H^{3}(k) \\
& \phi_{C} \quad \phi_{B} \\
& 0-\left[(a) \cup(b) \cup H^{1}(k(C))\right] \cap H_{n r}^{3}(k(C))-H_{n r}^{3}(k(C)) \stackrel{\bar{\phi}}{-} H_{n r}^{3}(k(B)) .
\end{aligned}
$$

The commuting triangle in this diagram gives an exact sequence

$$
0 \rightarrow \operatorname{Ker} \phi_{C} \rightarrow \operatorname{Ker} \phi_{B} \rightarrow \operatorname{Ker} \bar{\phi} \rightarrow \text { Coker } \phi_{C} .
$$

By a theorem of Arason (1.2), we have $\operatorname{Ker} \phi_{B}=(a) \cup(b) \cup H^{1}(k)$. The group $\operatorname{Ker} \phi_{C}$ is isomorphic to $\mathbb{Z} / 2\langle(a) \cup(b) \cup(c)\rangle(1.2)$. Further, by Theorem 1, the map $\phi_{C}$ is surjective if $(a) \cup(b) \cup(c) \cup(-1) \neq 0$ in $H^{4}(k)$ and Coker $\phi_{C}$ is isomorphic to $\mathbb{Z} / 2$ if $(a) \cup(b) \cup(c) \cup(-1)=0$ in $H^{4}(k)$. Using Lemma 3 in the latter case, we see that the map $\operatorname{Ker} \bar{\phi} \rightarrow$ Coker $\phi_{C}=\mathbb{Z} / 2$ is trivial. Thus, in both cases, the $\operatorname{map} \operatorname{Ker} \phi_{B} \rightarrow \operatorname{Ker} \bar{\phi}$ is surjective, and hence we have $\operatorname{Ker} \bar{\phi} \simeq \operatorname{Ker} \phi_{B} / \operatorname{Ker} \phi_{C}$. Therefore,

$$
\left[(a) \cup(b) \cup H^{1}(k(C))\right] \cap H_{n r}^{3}(k(C)) \simeq(a) \cup(b) \cup H^{1}(k) /(a) \cup(b) \cup(c),
$$

and the lemma is proved.

We now compute the group $H^{0}\left(V, \mathcal{H}^{4}\right)$. We recall some results of Szyjewski that are used in the computation and briefly mention the main steps involved in proving these results. Let $\mathcal{K}_{n}^{M}(1 \leq n \leq 3)$ denote the Milnor $K$-sheaf on a smooth integral variety $X / k[\mathrm{~K}]$. The homology of the complex (cf. $[\mathrm{K}]$ )

$$
C_{n}^{M}: 0 \rightarrow K_{n}^{M}(k(X)) \rightarrow \bigoplus_{x \in X^{(1)}} K_{n-1}^{M}(k(x)) \rightarrow \bigoplus_{x \in X^{(2)}} K_{n-2}^{M}(k(x)) \rightarrow \cdots
$$


computes the sheaf cohomology groups of $\mathcal{K}_{n}^{M}$. Here $X^{(i)}$ denotes the set of codimension $i$ points on $X$ and given $x \in X^{(i)}, k(x)$ denotes the residue field at $x$. The groups $K_{n}^{M}$ denote the Milnor $K$-groups of the fields. Recall that for any field $F$ (char $F \neq 2$ ), and $n \leq 3$, the norm residue homomorphism gives an isomorphism [Me], [Me-S1] [R]

$$
K_{n}^{M}(F) / 2 \simeq H^{n}(F)
$$

Thus, since $H^{n-1}(F)$ is generated by symbols for $n \leq 3$, using the description of torsion in $K_{2}$ for fields [Su, Theorem 1.8], we get a complex

$$
H^{n-1}(F) \stackrel{\{-1\}}{\rightarrow} K_{n}^{M}(F) \stackrel{2}{\rightarrow} K_{n}^{M}(F) .
$$

Here the first arrow maps $\left(a_{1}\right) \cup \ldots \cup\left(a_{n-1}\right)$ to the symbol $\left\{a_{1}, \ldots, a_{n-1},-1\right\}$, and this map is well-defined. This yields a complex (for $n \leq 3$ )

$$
C_{n-1}^{H} \stackrel{\{-1\}}{\rightarrow} C_{n}^{M} \stackrel{2}{\rightarrow} C_{n}^{M}
$$

where $C_{p}^{H}$ is the complex that computes the cohomology groups $H^{i}\left(X, \mathcal{H}^{p}\right)$ (cf. [B-O, Theorem 4.2]). Note that since the norm residue homomorphism $h_{n}$ : $K_{n}^{M}(F) / 2 \rightarrow H^{n}(F)$ is an isomorphism for $n \leq 3$, there is an isomorphism of complexes $C_{n}^{M} / 2 C_{n}^{M} \simeq C_{n}^{H}$ for $n \leq 3$. Using the excision sequence (cf. [K-Me, Proposition 2.3]), Szyjewski proves that for the open set $V$, the groups $H^{2}\left(V, \mathcal{K}_{3}^{M}\right)$ and $H^{3}\left(V, \mathcal{K}_{3}^{M}\right)$ are trivial [Sz1, Proposition 12.1.1 (c)]. Further, he proves (loc.cit.) that $H^{0}\left(V, \mathcal{K}_{3}^{M}\right) \simeq K_{3}^{M}(F)$ and $H^{1}\left(V, \mathcal{K}_{3}^{M}\right) \simeq D_{k}(\langle\langle a, b, c\rangle\rangle)$. Here, for a quadratic form $q$ over $k, D_{k}(q)$ represents the group of non-zero values represented by the quadratic form over $k$. The bicomplex associated to (15) for $V$ with $n=3$ gives a spectral sequence abutting to $H^{*}\left(V, \mathcal{H}^{3}\right)$. Using the above computations of the cohomology groups for $\mathcal{K}_{3}^{M}$, Szyjewski [Sz1, Proposition 12.2.1 (d)] proves that

$$
H^{1}\left(V, \mathcal{H}^{3}\right) \simeq D_{k}\langle\langle a, b, c\rangle\rangle /\left(D_{k}\langle\langle a, b, c\rangle\rangle\right)^{2} .
$$

We now prove

Lemma 5. Let $V$ be as before. Then the natural map $H^{4}(V) \rightarrow H^{0}\left(V, \mathcal{H}^{4}\right)$ is surjective.

Proof. As recalled above, the groups $H^{i}\left(V, \mathcal{K}_{3}^{M}\right)$ are trivial for $i=2$, 3. Note however that for $i=1,2$ these groups coincide with $H^{i}\left(V, \mathcal{K}_{3}\right)\left(\mathcal{K}_{3}\right.$ denotes the Quillen $K$-theory sheaf) [J-R, pp. 561-562]. Therefore the group $C H^{3}(V)$ is trivial. We have an exact sequence [CT1, p. 17]

$$
0 \rightarrow H^{2}\left(V, \mathcal{K}_{3}\right) / 2 \rightarrow H^{2}\left(V, \mathcal{H}^{3}\right) \rightarrow{ }_{2} C H^{3}(V) \rightarrow 0
$$

This sequence implies that $H^{2}\left(V, \mathcal{H}^{3}\right)$ is trivial. The Bloch-Ogus spectral sequence gives an exact sequence

$$
H^{4}(V) \rightarrow H^{0}\left(V, \mathcal{H}^{4}\right) \rightarrow H^{2}\left(V, \mathcal{H}^{3}\right)
$$

and the lemma follows.

Finally, we have the following:

Lemma 6. Assume that $(a) \cup(b) \cup(c) \cup(-1)$ is not zero in $H^{4}(k)$. Then the group $H^{0}\left(V, \mathcal{H}^{4}\right)$ is isomorphic to

$$
H^{4}(k) /(a) \cup(b) \cup(c) \cup H^{1}(F) \oplus D_{k}\langle\langle a, b, c,-1\rangle\rangle / D_{k}\langle\langle a, b, c\rangle\rangle
$$

with notation as above. 
Proof. The Hochschild-Serre spectral sequence for $V$ gives an exact sequence (cf. [Sz, Corollary 5.4.1])

$$
0 \rightarrow H^{4}(k) /(a) \cup(b) \cup(c) \cup(-1) \rightarrow H^{4}(V) \rightarrow L \rightarrow 0
$$

where $L \subseteq H^{1}(k)$ is the subgroup consisting of elements $(\lambda) \in k^{*} / k^{*^{2}}$ such that $(\lambda) \cup(a) \cup(b) \cup(c) \cup(-1)=0$ in $H^{5}(k)$.

Consider the filtration $H^{4}(V)^{(i)}$ on $H^{4}(V)$ associated to the Bloch-Ogus spectral sequence. Since $H^{2}\left(V, \mathcal{H}^{3}\right)$ is trivial, we have

$$
H^{0}\left(V, \mathcal{H}^{4}\right) \simeq H^{4}(V) / H^{4}(V)^{(1)} .
$$

Further, $H^{4}(V)^{(1)}$ is isomorphic to $H^{1}\left(V, \mathcal{H}^{3}\right)$ since $H^{4}(V)^{(2)} \simeq C H^{2}(V) / 2$ is trivial. By (16), the group $H^{1}\left(V, \mathcal{H}^{3}\right)$ is isomorphic to $D_{k}\langle\langle a, b, c\rangle\rangle /\left(D_{k}\langle\langle a, b, c\rangle\rangle\right)^{2}$. We analyse this group by considering the following filtration on $D_{k}\langle\langle a, b, c\rangle\rangle$ :

$$
\left(D_{k}\langle\langle a, b, c\rangle\rangle\right)^{2} \subseteq k^{*^{2}} \subseteq D_{k}\langle\langle a, b, c\rangle\rangle .
$$

The first quotient $D_{k}\langle\langle a, b, c\rangle\rangle / k^{*^{2}}$ is a natural subgroup of $H^{1}(k)$. The other quotient $k^{*^{2}} /\left(D_{k}\langle\langle a, b, c\rangle\rangle\right)^{2}$ fits in the exact sequence

$$
0 \rightarrow k^{*^{2}} /\left(D_{k}\langle\langle a, b, c\rangle\rangle\right)^{2} \rightarrow D_{k}\langle\langle a, b, c\rangle\rangle /\left(D_{k}\langle\langle a, b, c\rangle\rangle\right)^{2} \rightarrow D_{k}\langle\langle a, b, c\rangle\rangle / k^{*^{2}} \rightarrow 0 .
$$

Consider the natural surjection

$$
\begin{array}{cccc}
\pi: \quad k^{*} / D_{k}\langle\langle a, b, c\rangle\rangle & \rightarrow & k^{*^{2}} /\left(D_{k}\langle\langle a, b, c\rangle\rangle\right)^{2}, \\
(\lambda) & \mapsto & \left(\lambda^{2}\right) .
\end{array}
$$

Recall that the map $e_{4}$ is an isomorphism on Pfister forms, this being a consequence of Merkurjev's theorem and $e_{3}$ being well-defined (cf. [J-R, p. 555]). Under the assumption that $(a) \cup(b) \cup(c) \cup(-1)$ is not zero in $H^{4}(k)$, we see that the kernel of this map is isomorphic to $\mathbb{Z} / 2$, the class of $(-1)$ being the non-trivial element. The map

$$
\begin{array}{ccc}
\kappa: k^{*} / D_{k}\langle\langle a, b, c\rangle\rangle & \rightarrow & (a) \cup(b) \cup(c) \cup H^{1}(k) /(a) \cup(b) \cup(c) \cup(-1), \\
\lambda\left(\bmod D_{k}\langle\langle a, b, c\rangle\rangle\right) & \mapsto & (a) \cup(b) \cup(c) \cup(\lambda)(\bmod (a) \cup(b) \cup(c) \cup(-1)),
\end{array}
$$

along with the surjection $\pi$ thus gives an isomorphism

$$
k^{*^{2}} /\left(D_{k}\langle\langle a, b, c\rangle\rangle\right)^{2} \simeq(a) \cup(b) \cup(c) \cup H^{1}(k) /(a) \cup(b) \cup(c) \cup(-1) .
$$

Thus $H^{1}\left(V, \mathcal{H}^{3}\right)$ is (non-canonically) isomorphic to

$$
\begin{aligned}
H^{4}(V)^{(1)} & \simeq H^{1}\left(V, \mathcal{H}^{3}\right) \\
& \simeq(a) \cup(b) \cup(c) \cup H^{1}(k) /(a) \cup(b) \cup(c) \cup(-1) \oplus D_{k}\langle\langle a, b, c\rangle\rangle / k^{*^{2}} .
\end{aligned}
$$

Consider the two exact sequences

$$
0 \rightarrow H^{4}(k) /(a) \cup(b) \cup(c) \cup(-1) \rightarrow H^{4}(V) \rightarrow L \rightarrow 0,
$$

and

$$
\begin{aligned}
0 & \rightarrow(a) \cup(b) \cup(c) \cup H^{1}(k) /(a) \cup(b) \cup(c) \cup(-1) \\
& \rightarrow H^{4}(V)^{(1)} \rightarrow D_{k}\langle\langle a, b, c\rangle\rangle / k^{*^{2}} \rightarrow 0 .
\end{aligned}
$$


Recall that the first sequence follows from the Hochschild-Serre spectral sequence. The first two groups of the second exact sequence embed naturally into the corresponding groups of the first exact sequence. To see this, observe that

$$
\begin{aligned}
& H^{4}(V)^{(1)} \cap H^{4}(k) /(a) \cup(b) \cup(c) \cup(-1) \\
& \quad=(a) \cup(b) \cup(c) \cup H^{1}(k) /(a) \cup(b) \cup(c) \cup(-1) .
\end{aligned}
$$

This is because $\operatorname{Ker}\left(H^{4}(k) \rightarrow H^{4}(k(V))\right)=(a) \cup(b) \cup(c) \cup H^{1}(k)$ (1.2). Consider the induced map $D_{k}\langle\langle a, b, c\rangle\rangle / k^{*^{2}} \rightarrow L$. We claim that this map is also injective. To see this, consider the following commutative diagram

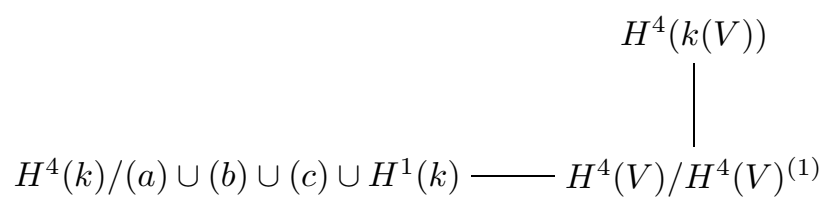

The vertical arrow and the slanted arrow are injective. This, along with the snake lemma applied to the two exact sequences above, proves the claim. Therefore we have an exact sequence

$$
0 \rightarrow H^{4}(k) /(a) \cup(b) \cup(c) \cup H^{1}(k) \rightarrow H^{4}(V) / H^{4}(V)^{(1)} \rightarrow L / D_{k}\langle\langle a, b, c\rangle\rangle \rightarrow 0
$$

and hence an isomorphism (non-canonical)

$$
H^{0}\left(V, \mathcal{H}^{4}\right) \simeq H^{4}(V) / H^{4}(V)^{(1)} \simeq H^{4}(k) /(a) \cup(b) \cup(c) H^{1}(k) \oplus L / D_{k}\langle\langle a, b, c\rangle\rangle .
$$

Suppose $(\lambda) \in L$. Then $(\lambda) \cup(a) \cup(b) \cup(c) \cup(-1)=0$ in $H^{5}(k)$. This implies that $e_{5}\langle\langle\lambda, a, b, c,-1\rangle\rangle=0$. By injectivity of $e_{5}$ on Pfister forms [J-R, Remark p. 555], we have $\langle\langle\lambda, a, b, c,-1\rangle\rangle=0$, which is equivalent to saying that $\lambda \in D_{k}\langle\langle a, b, c,-1\rangle\rangle$. We have therefore proved

$$
H^{0}\left(V, \mathcal{H}^{4}\right) \simeq H^{4}(k) /(a) \cup(b) \cup(c) \cup H^{1}(k) \oplus D_{k}\langle\langle a, b, c,-1\rangle\rangle / D_{k}\langle\langle a, b, c\rangle\rangle .
$$

Finally, we have

Corollary 6'. Suppose $k$ is a field such that $(a) \cup(b) \cup(c) \cup(-1) \neq 0$, and suppose $D_{k}\langle\langle a, b, c,-1\rangle\rangle=D_{k}\langle\langle a, b, c\rangle\rangle$. Then $H_{n r}^{4}(k(C)) \simeq H^{4}(k) /(a) \cup(b) \cup(c) H^{1}(k)$. In particular, the result is true for global fields.

Proof. The assumption on $k$ implies that $H^{0}\left(V, \mathcal{H}^{4}\right) \simeq H^{4}(k) /(a) \cup(b) \cup(c) H^{1}(k)$. From the excision sequence, we get an injection $H^{0}\left(C, \mathcal{H}^{4}\right) \hookrightarrow H^{0}\left(V, \mathcal{H}^{4}\right)$. Since $H^{4}(k)$ surjects onto $H^{0}\left(V, \mathcal{H}^{4}\right)$, the lemma follows. For global fields, we have isomorphisms $H^{i}(k) \stackrel{\cup(-1)}{\simeq} H^{i+1}(k)$ for $i \geq 3$, and the graded Witt ring of $k$ is isomorphic to the graded cohomology ring $[\overline{A E J}]$. This implies that the assumptions hold for global fields.

Remark. It does not seem straightforward to compute $H_{n r}^{4}(k(C))$ in a similar manner, as the sheaf cohomology groups $H^{p}\left(C, \mathcal{H}^{q}\right)$ are difficult to compute. One of the obstacles is the difficulty in deciphering the kernel of the differential $H^{0}\left(C, \mathcal{H}^{4}\right) \rightarrow$ $H^{2}\left(C, \mathcal{H}^{3}\right)$ in the Bloch-Ogus spectral sequence. 


\section{The CASE WHen $k$ IS A GLOBAL FIELD.}

In this section we assume that $k$ is a global field. Recall that we then have isomorphisms $H^{n}(k) \stackrel{\cup(-1)}{\simeq} H^{n+1}(k)$ for $n \geq 3$. If $k$ is totally imaginary, then $C$ is necessarily isotropic and hence we have isomorphisms $H^{i}(k) \simeq H_{n r}^{i}(k(C))$ for all $i$. We therefore assume that $k$ is not totally imaginary and $C / k$ is an anisotropic quadric. The natural map $H^{n}(k) \rightarrow H_{n r}^{n}(k(C))$ is surjective for $n \leq 4$. For $n \leq 2$, this is obvious from the Hochschild-Serre spectral sequence. For $n=3$, this follows from Theorem 1, noting that from the above remarks, $(a) \cup(b) \cup(c) \cup(-1)$ is not zero. For $n=4$, this follows from Corollary $6^{\prime}$. We prove the surjectivity for $n=5$, assuming certain results which we outline below. We remark that proofs for these results have been announced. For $n \geq 6$, we prove the surjectivity without any restrictions.

Let $k$ be a global field and $X / k$ be a smooth, projective, geometrically integral variety of dimension $d$. Given a place $v$ of $k$, let $k_{v}$ denote the completion. Let $k(X)$ (resp. $\left.k_{v}(X)\right)$ denote the function field of $X$ (resp. $X_{v}:=X \times k_{v}$ ). Consider the natural map (of Galois cohomology groups)

$$
H^{d+2}(k(X), \mathbb{Q} / \mathbb{Z}(d+1)) \rightarrow \prod_{v} H^{d+2}\left(k_{v}(X), \mathbb{Q} / \mathbb{Z}(d+1)\right)
$$

where $v$ varies over the places of $k$. The cohomological Hasse principle $H_{d}$ is said to hold if this map is injective. For $d=1$ this was proved by Kato, and Jannsen gave a proof for $d \leq 2$. Jannsen [Ja] has announced a proof for arbitrary $d$. Suppose that the norm residue homomorphism $h_{4}: K_{4}^{M}(F) / 2 \rightarrow H^{4}(F)$ is an isomorphism for any field $F$. From $H_{3}$, one can then deduce (cf. [Ra, p. 177]) that the natural map

$$
H^{5}(k(X)) \rightarrow \prod_{v} H^{5}\left(k_{v}(X)\right)
$$

where $v$ varies over all places, is injective. We now prove

Theorem 7. Let $C / k$ be as above. Assume that $h_{4}$ is an isomorphism for all fields $F$ and that the cohomological Hasse principle $H_{3}$ holds. Then the natural map $H^{5}(k) \rightarrow H_{n r}^{5}(k(C))$ is surjective. Further, there is an isomorphism $H_{n r}^{5}(k(C)) \simeq$ $\bigoplus_{v \mid \infty} H_{n r}^{5}\left(k_{v}(C)\right)$, the direct sum varying over the real places of $k$.

Remark. We remark that Rost [R1] has announced a proof that $h_{4}$ is an isomorphism. Jannsen [Ja] has announced a proof that $H_{d}$ is true for any $d$.

Proof. By our hypothesis, the natural map $H^{5}(k(C)) \rightarrow \prod_{v} H_{n r}^{5}(k(C))$ is injective, and hence, so is the restricted map $H_{n r}^{5}(k(C)) \rightarrow \prod_{v} H_{n r}^{5}\left(k_{v}(C)\right)$. For a finite place $v, C\left(k_{v}\right) \neq \emptyset$, since any quadratic form of dimension at least five is isotropic over a local field. Hence, we have isomorphisms $H^{n}\left(k_{v}\right) \simeq H_{n r}^{n}\left(k_{v}(C)\right)$ for all $n$. Since the 2-cohomological dimension $c d_{2}\left(k_{v}\right)$ is two, we have $H_{n r}^{n}\left(k_{v}(C)\right)=0=H^{n}\left(k_{v}\right)$ for $n \geq 3$. Similarly, if $k_{v}=\mathbb{C}$, the groups $H_{n r}^{n}\left(k_{v}(C)\right)$ are trivial for $n \geq 1$. If $v$ is a real place such that $C\left(k_{v}\right) \neq \emptyset$, then $H^{n}\left(k_{v}\right) \simeq H_{n r}^{n}\left(k_{v}(C)\right) \simeq \mathbb{Z} / 2$ for all $n$. If $v$ is a real place with $C\left(k_{v}\right)=\emptyset$, then $H_{n r}^{n}\left(k_{v}(C)\right)$ is trivial for $n \geq 4$, since $c d_{2}\left(k_{v}(C)\right)=3$. Therefore we have an injective map

$$
H_{n r}^{5}(k(C)) \hookrightarrow \prod_{v \mid \infty} H_{n r}^{5}\left(k_{v}(C)\right) .
$$


Consider the commutative diagram

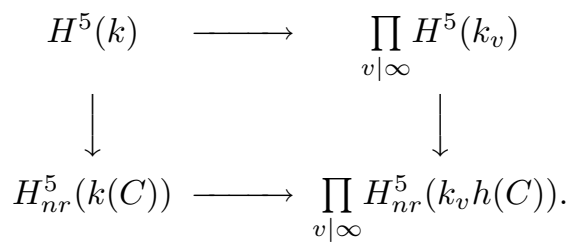

The top horizontal map is an isomorphism the right vertical map is surjective, and the lower horizontal map is injective. Hence the map $H^{5}(k) \rightarrow H_{n r}^{5}(k(C))$ is surjective and $H_{n r}^{5}(k(C)) \rightarrow \bigoplus_{v \mid \infty} H_{n r}^{5}\left(k_{v}(C)\right)$ is an isomorphism.

Theorem 8. Let $C / k$ be as above. Then the natural map $H^{i}(k) \rightarrow H_{n r}^{i}(k(C))$ is surjective for all $i \geq 6$.

Proof. Let $l=k(\sqrt{-1})$. Since $c d_{2}(l(C))=5$, the groups $H^{i}(k(C))$ and hence $H_{n r}^{i}(k(C))$ are (-1)-torsion free for $i \geq 6$ [AEJ, p. 29]. Recall that the (-1)-torsion subgroup of $H^{i}(k(C))$ is precisely the group $\operatorname{Ker}\left(H^{i}(k(C)) \rightarrow \mathcal{C}\left(\operatorname{Spec}_{r}(k(C), \mathbb{Z} / 2)\right)\right.$ [AEJ, p. 29]. Here $\mathcal{C}(A, B)$ denotes the set of continuous maps from a space $A$ into $B$ and Spec $_{r}$ denotes the real spectrum (cf. [C-R]). One checks that the (-1)-torsion subgroup of $H_{n r}^{i}(k(C))$ is the group $\operatorname{Ker}\left(H_{n r}^{i}(k(C)) \rightarrow \mathcal{C}\left(\operatorname{Spec}_{r} C, \mathbb{Z} / 2\right)\right)$. Since $k$ has only finitely many orderings corresponding to the real places, the natural map Spec $_{r} C \rightarrow \coprod_{v \mid \infty}$ Spec $_{r} C_{v}$ is a homeomorphism [C-R, Proposition 4.3]. Consider the commutative diagram

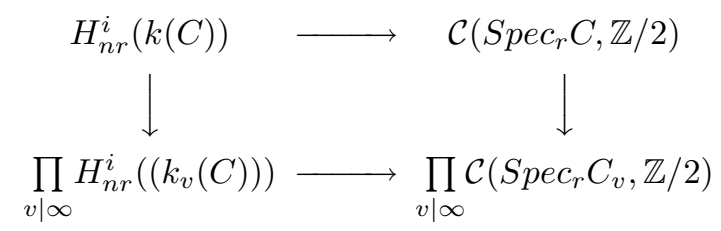

for $i \geq 6$. The right vertical map is an isomorphism and the top horizontal map is injective, since $H_{n r}^{6}(k(C))$ is $(-1)$-torsion-free. Therefore the left vertical map is injective for $i \geq 6$. Arguing as in the proof of Theorem 7, the surjectivity follows for $i \geq 6$.

Our next goal is to prove an analogous result for the groups $I_{n r}^{n}(k(C)), n \geq 5$. Before we prove the result, we need to compare the filtration of the Witt group with the cohomology groups. Towards this end, we note that for a field of transcendence degree three over a global field or a local field, the graded Witt ring is isomorphic to the graded cohomology ring once we use Rost's announced result that $\bar{e}_{4}$ is an isomorphism and $e_{5}$ is well-defined for all fields $F$. This follows by standard arguments of Arason-Elman-Jacob [AEJ3, Theorem 1.5]. We record this as

Theorem 9. Let $F$ be a field of transcendence degree at most three over a global (resp. local) field $k$. Suppose $\bar{e}_{4}$ is an isomorphism and $e_{5}$ is well-defined for $F$. Then the graded Witt ring is isomorphic to the graded cohomology ring of $F$.

We also remark that for a field of transcendence degree three over a real-closed field (resp. algebraically closed field), it is known that the graded Witt ring is isomorphic to the graded cohomology ring [AEJ2, Corollary 3]. We have 
Corollary 10. Let $C / k$ be as above. Assume that $\bar{e}_{4}$ is an isomorphism and $e_{5}$ is well-defined for the fields $k(C)$ and $k_{v}(C), v$ a finite place of $k$. Then the natural map $I^{n}(k) \rightarrow I_{n r}^{n}(k(C))$ is surjective for $n \geq 5$.

Proof. We first remark that an easy application of the 'Normality Theorem' due to Elman-Lam [E-L, Theorem 3.2] for $k$ shows that the map $I^{n}(k) \rightarrow \prod_{v} I^{n}\left(k_{v}\right)$ is surjective for $n \geq 3$. Since this map is injective, it is an isomorphism. Further, using Theorems 8 and 9 and arguing as in [AEJ2, Theorem 4], one sees that the map $I_{n r}^{n}(k(C)) \rightarrow \prod_{v} I_{n r}^{n}\left(k_{v}(C)\right)$ is injective for $n \geq 5$. Combining Theorem 9 with the Arason-Pfister Hauptsatz [Sc, p.156], one sees that the group $I_{n r}^{5}\left(k_{v}(C)\right)$ is trivial whenever $H_{n r}^{5}(k(C))$ is. Further, if $k_{v}$ is a real place with $C\left(k_{v}\right) \neq \emptyset$, then the specialisation map gives isomorphisms $I_{n r}^{n}\left(k_{v}(C)\right) \simeq I^{n}\left(k_{v}\right)$ for all $n$. Arguing exactly along the lines of the proof of Theorem 9 , we get the corollary.

\section{UNRAMIFIED WITT GROUPS OF ANISOTROPIC QUADRICS}

In this section, we prove that the unramified Witt group $W_{n r}(k(C))$ of the three dimensional quadric $C$ is a quotient of the Witt group $W(k)$ of $k$ under the assumption (A) below. We continue with the notation of earlier sections. Recall that (1.2) we have an exact sequence

$$
0 \rightarrow\langle\langle a, b\rangle\rangle W(k(C)) \rightarrow W(k(C)) \rightarrow W(k(C \times B))
$$

where the quadric surface $B$ is now considered as being defined over $k(C)$. Now $k(B \times C)=k(C \times B)$ is a pure transcendental extension of $k(B)(1.2)$, and the unramified Witt group remains invariant under pure transcendental extensions $[\mathrm{O}$, Proposition 7.2]. Hence, the restriction of (17) to the unramified subgroups gives an exact sequence

$$
0 \rightarrow[\langle\langle a, b\rangle\rangle W(k(C))] \cap W_{n r}(k(C)) \rightarrow W_{n r}(k(C)) \rightarrow W_{n r}(k(B))
$$

with notation as before. But since the quadratic form defining the anisotropic conic $X$ is a Pfister neighbour of the quadratic form defining $B$, we have [CT-S, Lemma 1.2] $W_{n r}(k(B)) \simeq W_{n r}(k(X)) \simeq W(k) /\langle\langle a, b\rangle\rangle W(k)$ (1.2). We now state the assumption we need.

(A): The subgroup $[\langle\langle a, b\rangle\rangle I(k(C))] \cap I_{n r}^{3}(k(C))$ is generated by elements of the form $\langle\langle a, b, f\rangle\rangle \in I_{n r}^{3}(k(C))$, where $I_{n r}^{3}(k(C))=W_{n r}(k(C)) \cap I^{3}(k(C))$.

This assumption is to be viewed as a "quadratic-form" version of Lemma 3 . We use this to prove the following:

Theorem 11. Let $C / k$ be the smooth projective anisotropic quadric of dimension three defined by the quadratic form $\langle\langle a, b\rangle\rangle \perp\langle-c\rangle$. Assume $(\mathbf{A})$ is true. Then the unramified Witt group $W_{n r}(k(C))$ is isomorphic to $W(k) /\langle\langle a, b, c\rangle\rangle W(k)$.

Proof. We use the exact sequence (17), observing that by the remarks above, we have $W_{n r}(k(B)) \simeq W(k) /\langle\langle a, b\rangle\rangle W(k)$. Considering the natural maps, we have a commuting diagram with exact row as follows:

$$
\begin{aligned}
& W(k) \\
& \eta_{C} \quad \eta_{B} \\
& 0-[\langle\langle a, b\rangle\rangle W(k(C))] \cap W_{n r}(k(C))-W_{n r}(k(C))-\bar{\eta} \quad W(k) /\langle\langle a, b\rangle\rangle W(k) .
\end{aligned}
$$


Here $\eta_{C}$ and $\eta_{B}$ denote the natural maps, and clearly $\bar{\eta}$ is surjective. To prove that $\eta_{C}: W(k) \rightarrow W_{n r}(k(C))$ is surjective, note that by (19), it suffices to prove the following: Given an element $\left\langle q_{1}\right\rangle$ in $[\langle\langle a, b\rangle\rangle W(k(C))] \cap W_{n r}(k(C))$, there is an element $\left\langle\tilde{q}_{1}\right\rangle$ in $W(k)$ such that

$$
\eta_{C}\left\langle\tilde{q}_{1}\right\rangle=\left\langle q_{1}\right\rangle .
$$

We now proceed to prove this. Consider the ideal $I(k(C)) \subseteq W(k(C))$ of even dimensional forms. Then we have $W(k(C)) / I(k(C)) \simeq \mathbb{Z} / 2$, with the non-trivial element being the class $\langle 1\rangle$, which clearly belongs to $W(k)$. Thus, it suffices to prove $(*)$ for elements which are in the subgroup $[\langle\langle a, b\rangle\rangle I(k(C))] \cap W_{n r}(k(C))$. By assumption, this group is generated by elements of the form $\langle\langle a, b, f\rangle\rangle$. Since the homomorphisms $e_{3}$ and $e_{2}$ are well-defined, (1.2), we have $e_{3}(\langle\langle a, b, f\rangle\rangle)=$ $(a) \cup(b) \cup(f) \in H_{n r}^{3}(k(C))(1.2)$. Further, it is clear that the element $(a) \cup(b) \cup(f) \in$ $\left[(a) \cup(b) \cup H^{1}(k(C))\right] \cap H_{n r}^{3}(k(C))$. By Lemma 4, we have

$$
(a) \cup(b) \cup(f)=(a) \cup(b) \cup(d)+(a) \cup(b) \cup(c), \quad d \in k^{*},
$$

and this implies that

$$
(a) \cup(b) \cup(f)=(a) \cup(b) \cup(e) \in H^{3}(k), \quad e \in k^{*} .
$$

Consider the Pfister forms $\langle\langle a, b, f\rangle\rangle$ and $\langle\langle a, b, e\rangle\rangle$. These elements belong to the subgroup $[\langle\langle a, b\rangle\rangle I(k(C))] \cap W_{n r}(k(C))$. Further, by (20), we have

$$
e_{3}\langle\langle a, b, f\rangle\rangle=(a) \cup(b) \cup(f)=(a) \cup(b) \cup(e)=e_{3}\langle\langle a, b, e\rangle\rangle .
$$

Since $e_{3}$ is injective on Pfister forms [A2, Proposition 2], we have $\eta_{C}\langle\langle a, b, e\rangle\rangle=$ $\langle\langle a, b, f\rangle\rangle$, and $(*)$ is proved.

Thus the natural map $\eta_{C}: W(k) \rightarrow W_{n r}(k(C))$ is surjective. Further, since the form defining $C$ is a Pfister neighbour of the three-fold Pfister form $\langle\langle a, b, c\rangle\rangle$, by (1.2) we have,

$$
\operatorname{Ker}\left(W(k) \rightarrow W_{n r}(k(C))\right)=\operatorname{Ker}(W(k)) \rightarrow W(k(C))=\langle\langle a, b, c\rangle\rangle W(k) .
$$

Hence $W_{n r}(k(C)) \simeq W(k) /\langle\langle a, b, c\rangle\rangle W(k)$, and the theorem is proved.

In general, we have

Theorem 12. Let $C / k$ be as above. Then the natural map $W(k) \rightarrow W_{n r}(k(C))$ gives a surjection $W(k) / I^{4}(k) \rightarrow W_{n r}(k(C)) / I_{n r}^{4}(k(C))$.

Proof. Arguing as in the proof of Theorem 11, this is immediate from Theorem 1, since $\bar{e}_{3}$ is a well-defined isomorphism (1.2).

Assuming that $k$ is a global field, we have

Theorem 13. Let $k$ be a global field and $C$ an anisotropic three dimensional quadric over $k$ defined by $\langle\langle a, b\rangle\rangle \perp\langle-c\rangle$. Assume that the norm residue homomorphism $h_{4}$ is an isomorphism for all fields and that $e_{5}$ is well-defined. Under the assumption that the cohomological Hasse principle $\mathrm{H}_{3}$ holds, there is an isomorphism

$$
W_{n r}(k(C)) \simeq W(k) /\langle\langle a, b, c\rangle\rangle W(k) .
$$

Remark. We remark that Rost [R1] has announced a proof that $h_{4}$ is an isomorphism and $e_{5}$ is well-defined. Jannsen [Ja] has announced a proof that $H_{d}$ holds for all $d$. 
Proof. The initial steps in the proof are analogous to those in the proof of Theorem 11. We now prove $(*)$ (cf. proof of Theorem 11) without assuming (A). As before, we reduce to proving $(*)$ for elements in the subgroup $[\langle\langle a, b\rangle\rangle] I(k(C)) \cap W_{n r}(k(C))$. Let $q_{1}$ be an element of this group. Then, $q_{1} \in I_{n r}^{3}(k(C))$ and $e_{3}\left(q_{1}\right) \in H_{n r}^{3}(k(C))$. By Theorem 1, $e_{3}\left(q_{1}\right)=(a) \cup(b) \cup(e),(e) \in H^{1}(k)$. Thus $\langle\langle a, b, e\rangle\rangle$ and $q_{1}$ map to the same element under $e_{3}$, and hence $q_{1}=\langle\langle a, b, e\rangle\rangle \perp q_{2}$ where $q_{2} \in I_{n r}^{4}(k(C))$. Again, $e_{4}\left(q_{2}\right) \in H_{n r}^{4}(k(C))$ can be lifted to a symbol in $H^{4}(k)$ under the natural map $H^{4}(k) \rightarrow H_{n r}^{4}(k(C))$, which is surjective by Corollary $6^{\prime}$. Therefore $q_{2}=\tilde{q_{2}} \perp q_{3}$ where $\tilde{q_{2}} \in I^{4}(k)$ and $q_{3} \in I_{n r}^{5}(k(C))$. By Corollary 10 , there exists $\tilde{q_{3}} \in I^{5}(k)$ such that $\tilde{q}_{3}$ maps to $q_{3}$ under the natural map $I^{5}(k) \rightarrow I_{n r}^{5}(k(C))$, and hence $(*)$ is proved.

We have the following corollary.

Corollary 14. With hypotheses as in Theorem 13, any unramified quadratic form over $k(C)$ is defined over $k$.

Proof. Let $q$ be an unramified quadratic form over $k(C)$. Without loss of generality, we may assume that $q$ is anisotropic. By a result of Elman-Lam-Wadsworth [ELW, Corollary 6.4], the field $k(C)$ is an excellent extension of $k$. The corollary now follows from Theorem 13.

Note added in proof. By the recent results of Voevdosky, the norm-residue maps $h_{n}$ are isomorphisms for all $n$. In particular, the maps $\bar{e}_{n}$ are well-defined isomorphisms for all $n$.

\section{REFERENCES}

[A] Arason, J. Kr., Cohomologische Invarianten quadratischer Formen, J. Algebra 36 (1975), 448-491. MR 52:10592

[A2] Arason, J. Kr., A proof of Merkurjev's Theorem, Canadian Math. Soc. Conf. Proc., vol. 4, Amer. Math. Soc., Providence, RI, 1984, pp. 121-130. MR 86f:11029

[AEJ] Arason, J. Kr., Elman, R., Jacob, B., Graded Witt ring and Galois cohomology, Canadian Math. Soc. Conf. Proc. vol. 4, Amer. Math. Soc., Providence, RI, 1984, pp. 17-50. MR 86g:11020

[AEJ2] Arason, J. Kr., Elman, R., Jacob, B., Fields of cohomological 2-dimension three, Math. Ann. 274 (1986), 649-657. MR 87m:12006

[AEJ3] Arason, J. Kr., Elman, R., Jacob, B., Graded Witt ring and Galois cohomology II, Transactions Amer. Math. Soc. 314 (1989), 745-780. MR 90a:11043

[B-O] Bloch, S., Ogus, A., Gersten's conjecture and the homology of schemes, Ann. Sc. Éc. Norm. Supér (4) (1974), 181-202. MR 54:318

[CT] Colliot-Thélène, J.-L., Birational invariants, purity and the Gersten conjecture, Proc. Sym. Pure Math 58 (I) (1995), 1-64. MR 96c:14016

[CT1] Colliot-Thélène, J.-L., Cycles algébriques de torsion et K-théorie algébrique, Lecture notes in Mathematics 1553 (1992), 1-49. MR 96h:14006

[CT-O] Colliot-Thélène, J.-L., Ojanguren, M., Variétés unirationnelles non rationnelles: audelà de l'exemple d'Artin et Mumford, Invent. Math. 97 (1989), 141-158. MR 90m:14012

[CT-S] Colliot-Thélène, J.-L., Sujatha, R., Unramified Witt groups of real anisotropic quadrics, Proc. Sym. Pure Math 58 (II) (1995), 127-147. MR 96e:19009

[E-L] Elman, R., Lam, T.-Y., Pfister forms and K-theory of fields, Jour. Alg 23 (1972), 181213. MR 46:1882

[ELW] Elman, R., Lam, T. Y., Wadsworth, A. R., Function fields of Pfister forms, Invent. Math. 51 (1979), 61-75. MR 80m:10017

[J-R] Jacob, B., Rost, M., Degree four cohomological invariants for quadratic forms, Invent. Math 96 (1989), 551-570. MR 90g:11044 
[Ja] Jannsen, U., Cohomological Hasse principles, Handwritten notes.

[Ka] Kahn, B., Lower H-cohomology of higher dimensional quadrics, Arch. Math. (Basel) 65 (1995), 244-250. MR 97a:14017

[K-Me] Karpenko, N.A., Merkurjev, A.S., Chow groups of projective quadrics, Leningrad Math. Jour. 2 (1991), 655-671. MR 91i: 14003

[K] Kato, K., Milnor K-theory and the Chow group of zero cycles, Contemp. Math. 55 (1986), 241-253. MR 88c:14012

[Me] Merkurjev, A. S., On the norm residue symbol of degree 2, Soviet Math. Dokl. 24 (1981), 546-551. MR 83h:12015

[Me-S1] Merkurjev, A. S., Suslin, A. A., The norm-residue homomorphism in degree three, Math. USSR. Izv. 36 (1991), 349-367. MR 91f:11083

[Mi] Milne, J. S., Étale Cohomology, Princeton University Press, 1980. MR 81j:14002

[O] Ojanguren, M., The Witt group and the problem of Lüroth, ETS Editrice Pisa, Pisa, 1991. MR 91m:11031

[P] Parimala, R., Witt groups of conics, elliptic and hyperelliptic curves, Jour. Number Theory 28 (1988), 69-93. MR 89a:14028

[P2] Parimala, R., Witt groups of affine three-folds, Duke Math. J. 57 (1988), 947-954. MR 90a:14006

[Pf] Pfister, A., Multiplikative quadratische Formen, Arch. Math. 16 (1965), 363-370. MR 32:2408

[Ra] Raskind, W., Abelian class field theory of arithmetic schemes, Proc. Sym. Pure Math 58 (I) (1995), 85-187. MR 96b:11089

[R] Rost, M., Hilbert Theorem 90 for $K_{3}$ for degree two extensions, preprint, Regensburg, 1986.

[R1] Rost, M., Talk at the Ascona Conference on Quadratic Forms, 1991.

[Sc] Scharlau, W., Quadratic and Hermitian forms, Grundlehren der Mathematischen Wissenschaften 270, Berlin, Heidelberg, New York: Springer, 1985. MR 86k:11022

[Su] Suslin, A. A., Torsion in $K_{2}$ of fields, $K$-theory 1 (1987), 5-29. MR 89a:11123

[Sz] Shyevski [Szyjewski], M., The fifth invariant of quadratic forms, Leningrad Math Jour. 2 (1991), 179-198. MR 91d:11040

[Sz1] Shyevski [Szyjewski], M., Algebraic K-theory and quadratic forms (in Russian), Dissertation, Leningrad State University (1989).

Department of Mathematics, Ohio State University, 231 W 18th Avenue, Columbus, OHIO 43210

Permanent address: School of Mathematics, Tata Institute of Fundamental Research, Homi Bhabha RoAd, Bombay 400 005, India

E-mail address: sujatha@math.tifr.res.in 